\title{
The Hospitalized Patient with Interstitial Lung Disease: A Hospitalist Primer
}

\author{
Erica D. Farrand, MD*, Rupal J. Shah, MD, Harold R. Collard, MD
}

Department of Medicine, School of Medicine, University of California San Francisco, San Francisco, California.

Interstitial lung disease (ILD) is a diverse group of disorders typically with insidious onset. Diagnosis and management largely occur in the outpatient setting; however, ILD can present acutely necessitating hospitalization. Effective inpatient management requires the clinician to establish an accurate diagnosis and understand the natural history and treatment responsiveness of each ILD subtype. We propose a general framework for approaching the evaluation of hospitalized patients with ILD, and provide focused guidance on key inpatient diagnostic and management decisions. Journal of Hospital Medicine 2017;12:580-584. (C) 2017 Society of Hospital Medicine
Interstitial lung disease (ILD) encompasses a diverse group of disorders that cause inflammation and fibrosis of the lung parenchyma. The clinical manifestations, disease course, management and prognosis of ILD vary depending on the underlying subtype, making accurate classification and diagnosis an important initial step. While a comprehensive list of ILD contains dozens of disorders, the majority of patients will fall into 1 of 3 categories: exposure-related ILD, connective tissue disease-related ILD (CT-ILD), and the idiopathic interstitial pneumonias (Table).

An essential first step in the evaluation of every hospitalized patient with ILD is establishing a diagnosis. A common mistake among clinicians who diagnose patients with ILD is not realizing that ILD is a collection of diseases with different etiologies, natural histories, and treatments. A careful evaluation should be performed in every hospitalized patient with ILD to ensure an accurate diagnosis, ideally in the context of a multidisciplinary conference with pulmonary, radiology, pathology, and other specialties, as appropriate. A multidisciplinary panel of the American Thoracic Society/ European Respiratory Society recently published a revised classification of ILD based on a combination of clinical, radiologic, and histopathologic findings, which may aid in refining the diagnosis. ${ }^{1}$

There are 3 main scenarios in which the hospital physician will encounter patients with ILD.

Acute presentation of new-onset disease. While many ILDs present insidiously, some cases present acutely and require hospitalization. The most common of these are acute hypersensitivity pneumonitis (HP), CT-ILD (in particular, myositis-related and systemic lupus erythematosus-related), drug-induced ILD (eg, amiodarone, nitrofurantoin), cryptogenic organizing pneumonia (COP), acute eosinophilic

*Address for correspondence and reprint requests: Erica D. Farrand, MD, 505 Parnassus Avenue, Box 0111, San Francisco, CA, 94143; Telephone: 415476-0735; Fax: 415-502-1321; E-mail: erica.farrand@ucsf.edu

Received: December 12, 2016; Revised: January 31, 2017; Accepted: February 5, 2017

2017 Society of Hospital Medicine DOI 10.12788/jhm.2774 pneumonia (AEP), and acute interstitial pneumonia (AIP).

Acute presentation of established (chronic) disease. $\mathrm{Pa}$ tients with chronic forms of ILD can present to the hospital with an acute exacerbation of disease. This can be caused by extra-parenchymal complications, including pulmonary embolism, pneumothorax, and pleural effusion; parenchymal complications such as infectious pneumonia, aspiration pneumonitis, and congestive heart failure; or without an identifiable cause. This latter presentation is most commonly seen in idiopathic pulmonary fibrosis (IPF).,3

Elective hospitalization for diagnostic surgical lung biopsy. Patients with ILD may be hospitalized electively for a laparoscopic surgical lung biopsy as part of their diagnostic evaluations.

Physicians caring for a hospitalized ILD patient must be familiar with the clinical presentations, diagnostic approach, medical management, and outpatient follow-up recommended in these 3 settings. We will summarize these areas and provide answers to commonly encountered clinical questions in the hospitalized patient with ILD.

\section{CLINICAL PRESENTATION}

Acute onset (or worsening) of dyspnea is the primary presenting symptom in most patients hospitalized for ILD. This symptom should be further characterized by assessing the degree of dyspnea and the extent of exercise limitation, as both impact overall disease severity and prognosis. ${ }^{4}$ Cough is the second most common symptom, and can be nonproductive, as is common in IPF, or be associated with secretions if parenchymal infection or acute bronchitis is present. ${ }^{5}$ Pleuritic chest pain, pleural effusion, and/or the presence of extrapulmonary features, including dysphagia, joint pain and swelling, or cutaneous thickening may suggest the presence of a CT-ILD. Because most forms of ILD present with only nonspecific symptoms, a careful history and physical examination are essential.

\section{DIAGNOSIS}

History

A comprehensive patient history is the backbone of diagnosing any ILD. History-taking should focus on severity and 
TABLE. Clinical Classification of Interstitial Lung Diseases

\begin{tabular}{lll}
\hline Exposure-Related & Connective Tissue Diseases & Idiopathic Interstitial Pneumonias \\
\hline Occupational & Scleroderma & Idiopathic pulmonary fibrosis \\
Environmental & Rheumatoid arthritis & Nonspecific interstitial pneumonia \\
Avocational & Sjögren's syndrome & Acute interstitial pneumonia \\
Medication & Systemic lupus erythematosus & Cryptogenic organizing pneumonia \\
Hypersensitivity Pneumonitis & Mixed connective tissue disease & Respiratory bronchiolitis interstitial lung disease Lymphangioleiomyomatosis \\
& Polymyositis & Desquamative interstitial pneumonia \\
& Dermatomyositis & \\
\hline
\end{tabular}

temporal progression of symptoms, presence of pre-existing systemic conditions associated with ILD, symptoms of extrapulmonary disease, and exposures to substances that can cause pulmonary injury, including a detailed history of occupations and hobbies, medications, smoking, and familial lung disease. ${ }^{6-9}$ Physicians must try to exclude other diagnoses that could result in a similar acute presentation, including congestive heart failure and infection. Considering the complex and extensive recommended history-taking, physicians may find it helpful to use a standardized questionnaire, as provided by the American College of Chest Physicians. ${ }^{10}$

\section{Laboratory Testing}

All patients presenting to the hospital with a suspected ILD should undergo careful assessment for the presence of connective tissue disease, including patients without clear symptoms because ILD can be the presenting manifestation. We routinely test for antinuclear antibody titer and pattern, rheumatoid factor, anticyclic citrullinated peptide, creatinine kinase, and aldolase as the initial screening panel in most patients, with further testing directed by the findings on history and physical examination. Pulmonary function tests are used routinely to monitor disease progression in the outpatient setting; however, in the hospitalized ILD patient, they are often difficult to perform and have no real diagnostic value. Similarly, arterial blood gas is not routinely used as part of the initial inpatient evaluation.

\section{Imaging}

All hospitalized patients with a known or suspected ILD should undergo chest imaging, assuming they are stable enough to do so. While the chest radiograph can provide a low-cost initial assessment of the degree of lung involvement and presence of accompanying abnormalities, computed tomography (CT) scanning is the diagnostic test of choice. ${ }^{11}$ The pattern and distribution of abnormalities on CT scan can greatly assist with the differential diagnosis in patients presenting with a new ILD, while the presence and pattern of new opacities superimposed on chronic changes can inform the differential and the prognosis of an ILD exacerbation. ${ }^{12}$ High-resolution CT provides the most sensitive imaging modality for diffuse ILD. The addition of prone and expiratory images are helpful in differentiating mild lung disease from atelectasis and detecting air trapping, respec- tively. ${ }^{13}$ However, since pulmonary embolism is a common extraparenchymal finding routinely considered in the differential of a patient presenting with a known or suspected ILD, physicians should consider ordering a CT pulmonary angiogram with additional high-resolution images. Most important, radiographic evaluation should include a review of all available prior chest imaging to assess both the tempo and the nature of radiographic findings.

\section{Bronchoscopy}

Bronchoscopy (with bronchoalveolar lavage [BAL], transbronchial lung biopsy [TBLB] and/or transbronchial needle aspiration [TBNA]) is not a routinely used diagnostic tool in the hospitalized ILD patient. However, it should be considered in certain circumstances. ${ }^{7}$ Cell count and differential can be helpful in diagnosing AEP (greater than 40\% eosinophilia) or acute HP (greater than 50\% lymphocytosis), while the addition of microbiologic and cytologic analysis can assist with the diagnosis of infectious etiologies (including pneumocystis pneumonia) or malignancy. ${ }^{14,15}$ Bronchoscopy with BAL has limited sensitivity for many infections and the procedure is associated with a small risk of worsened hypoxemia. Transbronchial lung biopsy, and to a lesser extent TBNA, carry the added risk of pneumothorax and bleeding. In the majority of cases of ILD, TBLB and TBNA have limited diagnostic utility given the small amount of lung tissue sampled. In cases of suspected IPF, where the identification of the histologic pattern is needed for definitive diagnosis, tissue from TBLB cannot be used to make a conclusive diagnosis. ${ }^{16,17}$ However, both TBNA and TBLB are useful in the diagnosis of granulomatous disorders, such as sarcoidosis, where the diagnostic yield ranges from $80 \%$ to $90 \%$ and $50 \%$ to $75 \%$, respectively. ${ }^{18,19}$

A newer bronchoscopic approach to sampling the lung using a bronchoscopically-placed cryoprobe (termed transbronchial cryobiopsy) has uncertain diagnostic utility and safety in the acute setting. This procedure involves intubation, sedation, and bronchoscopy allowing for the passage of an endobronchial cryoprobe through the bronchoscope and into the periphery of the lung. Several cryobiopies are generally taken from the same pulmonary subsegment. Despite a large number of recent publications on this topic, none of them have provided a clear sense of the diagnostic yield and safety. ${ }^{20,21}$ Transbronchial cryobiopsy remains a highly 
controversial procedure in the clinical setting, and we would not recommend its use until further data are available. ${ }^{22}$

\section{Surgical Lung Biopsy}

In the outpatient setting, a surgical lung biopsy is often useful when the ILD diagnosis cannot be made from the clinical context and imaging. However, patients presenting with acute respiratory failure from ILD are at greatly increased risk of complications from nonelective biopsy including pneumothorax, hemothorax, acute exacerbation of ILD, ICU admission, mechanical ventilation, and in-hospital mortality. ${ }^{23,24}$ Acute histological findings can also make it difficult to appreciate the underlying pattern of fibrosis, reducing the diagnostic utility. ${ }^{25-27}$ In our experience, surgical lung biopsy rarely alters the treatment of ILD patients presenting in acute respiratory failure. We believe that surgical lung biopsy should be reserved for the rare hospitalized patients in whom the clinician believes the results would clearly change management and that the substantial risk is worth taking. ${ }^{5,28}$

\section{INPATIENT MANAGEMENT}

The inpatient management of ILD is a large topic and difficult to comprehensively cover in a single review. Therefore, in this section, we will review 6 key management questions that address both general and specific treatment decisions that frequently arise in the care of hospitalized ILD patients (Figure).

\section{When should hospitalized ILD patients be treated with antibiotics?}

Infection and acute presentations of ILD have many similar clinical and radiographic features, making it difficult to dis- tinguish between the two, or exclude infection as the causative role in an acute exacerbation. ${ }^{2}$ In many ILD patients, the risk of infection is higher than in the general population, due to the acute and chronic use of immunosuppression. Until firm guidelines on the use of antibiotics in hospitalized patients with acute respiratory symptoms are available, we recommend considering the empiric use of antibiotics in ILD patients in respiratory failure, in addition to a thorough infectious workup.

\section{When should hospitalized ILD patients be treated with corticosteroids?}

Clinical experience supports the use of corticosteroids in the acute management of most rapidly progressive ILDs presenting with respiratory failure, including AEP, COP, acute HP, drug-induced ILD, and some cases of CT-ILD. Patients with AEP tend to respond rapidly to corticosteroids. In a series of 137 patients with AEP, 127 (92\%) received corticosteroids, with defervescence and improved dyspnea within 48 to 72 hours and resolution of all symptoms after a median of 7 (4 to 10 ) days. ${ }^{29}$ Cryptogenic organizing pneumonia is similarly corticosteroid-responsive, with patients typically started on doses of $1 \mathrm{mg} / \mathrm{kg}$ of prednisone followed by a slow taper due to the risk of relapse. ${ }^{30}$ For the majority of acute CT-ILD, oral prednisone is the initial treatment, often in combination with a second immunosuppressive agent such as mycophenolate.

No proven therapies are available for acute exacerbations of IPF (AE-IPF), including the use of corticosteroids. The most recent international guidelines on the management of AE-IPF conditionally recommends the use of cortico-

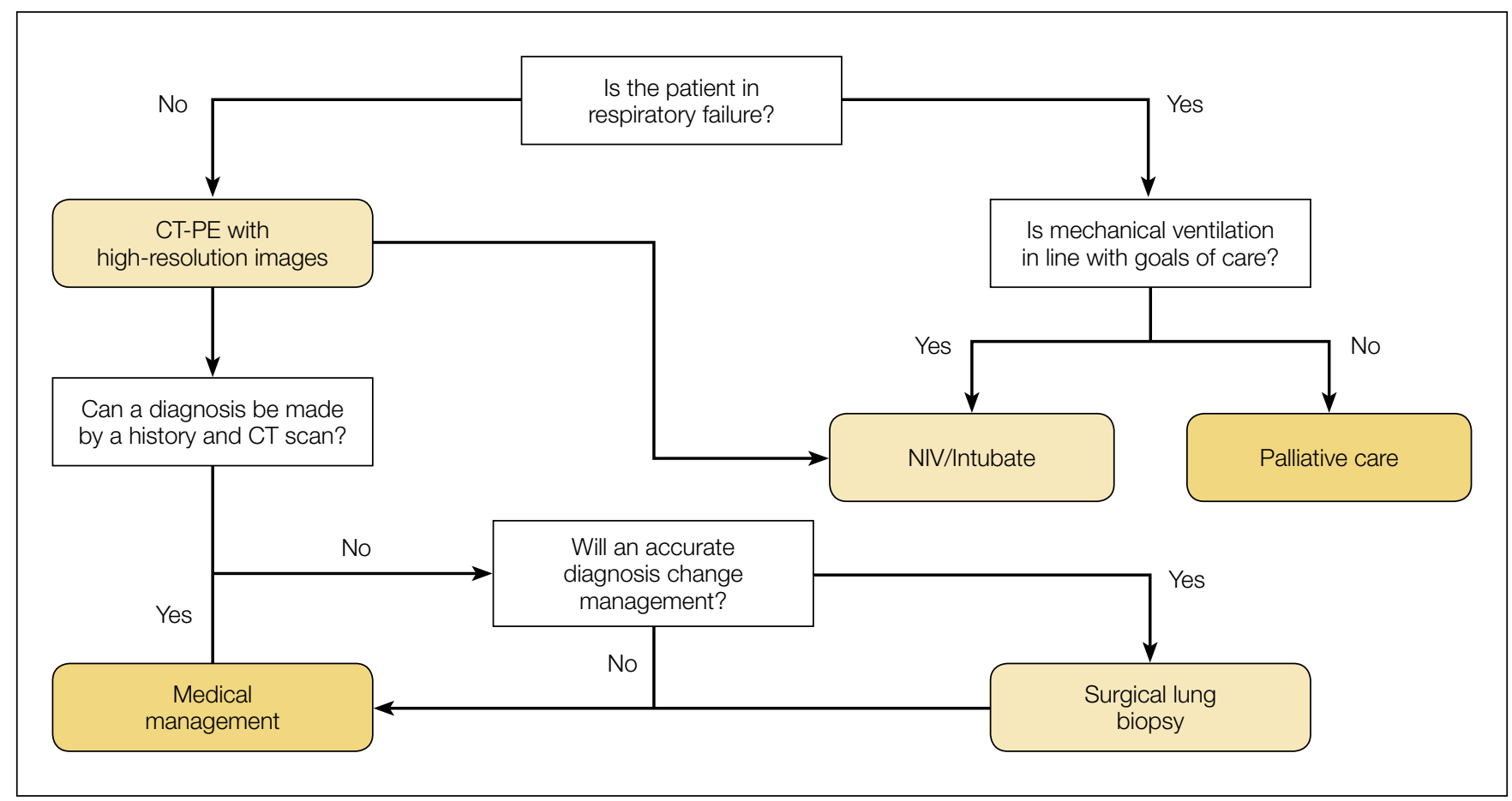

FIG. Proposed framework for the inpatient evaluation of hospitalized ILD patients.

NOTE: Abbreviation: CT, computed tomography; ILD, interstitial lung diseases; NIV, noninvasive ventilation; PE, pulmonary embolism. 
steroids, although this recommendation is largely based on anecdotal reports and clearly states that randomized studies are needed. ${ }^{3}$ When corticosteroids are used, we recommend high doses (eg, 1 to $2 \mathrm{mg} / \mathrm{kg}$ of prednisone) with close clinical monitoring. Consider stopping corticosteroids after 3 to 5 days if there is no evidence of clinical improvement. Prolonged courses of corticosteroids should be avoided.

\section{What additional pharmacologic therapies should be considered in the treatment of hospitalized ILD patients?}

Immunomodulators. Patients presenting acutely with a new-onset ILD or with an acute exacerbation of a chronic ILD often receive corticosteroids, sometimes in concert with an immunomodulator. This is most commonly seen in the acute management of CTD- ILD and in chronic HP, where mycophenolate mofetil, and to a lesser extent, cyclophosphamide and azathioprine for CT-ILD are used in combination with corticosteroids. The rationale for this is both therapeutic synergy and a desire to limit the long-term exposure to corticosteroids. Similarly, multiple observational cohort studies have investigated the role of combination or tandem immunosuppression in the treatment AE-IPF. Although cyclosporine, cyclophosphamide, azathioprine, rituximab and tacrolimus have all been studied, their efficacy remains uncertain. ${ }^{3}$ Until these therapies are better studied, they have no routine role in the management of AE-IPF.

Antifibrotics. Nintedanib and pirfenidone are 2 antifibrotic agents approved for the treatment of IPF. Clinical trials suggest that, in addition to slowing disease progression, these therapies may help prevent AE-IPF. The data are most robust in studies of nintedanib. A phase 2 trial with 432 subjects demonstrated a delay in time to the first investigator-reported acute exacerbation. ${ }^{31}$ Two follow-up phase 3 trials showed a reduction in centrally adjudicated AE-IPF in the pooled nintedanib groups compared to placebo. ${ }^{32}$ An initial phase 2 trial of pirfenidone showed a reduction in acute exacerbations in patients on pirfenidone, but this finding was not replicated in follow-up studies. ${ }^{33-35}$ Because of their potential role in preventing acute exacerbations and emerging evidence to suggest that continuation of antifibrotics may lead to better outcomes during an acute exacerbation, these drugs should not generally be stopped during a hospitalization for ILD. However, no evidence supports their initiation during acute exacerbations, and we do not recommend starting antifibrotics in the hospitalized setting for newly diagnosed patients. Starting and stopping antifibrotics should be reserved for outpatient management.

\section{When should noninvasive and mechanical ventilation be considered?}

We recommend carefully considering the use of noninvasive ventilation (NIV) and intubation in every ILD patient in respiratory distress, as an acutely reversible process may be present. In patients requiring mechanical ventilation, every effort should be made to minimize potential damage by reducing the fraction of inspired oxygen (to prevent potential hyperoxic injury) and reducing tidal volumes (to minimize barotrauma). Patients with a chronic ILD, particularly IPF, who require NIV or mechanical ventilation will generally have poor outcomes.

Studies suggest that NIV prevents mechanical ventilation in only the minority of patients presenting with an AE-IPF and is associated with high in-hospital mortality and a median survival following hospital discharge of only 60 days. ${ }^{36-38}$ The majority of patients with IPF requiring mechanical ventilation will not survive the intensive care unit. In a series of 23 patients presenting with acute respiratory failure and IPF, 22 of the 23 patients died while receiving mechanical ventilation, with a median survival of 3 days. In a more recent study of 34 patients with acute respiratory failure and IPF, 15 subjects underwent mechanical ventilation with an in-hospital mortality rate of $100 \% .{ }^{39}$ Given the overall poor survival associated with AE-IPF, mechanical ventilation should be carefully considered with the patient and family as part of an overall goals-of-care conversation prior to initiation.

\section{When should hospitalized ILD patients be referred for inpatient lung transplant evaluation?}

A subset of hospitalized patients with ILD will not respond to supportive and pharmacologic care, particularly those with advanced lung fibrosis. In these cases, lung transplantation may be the only remaining treatment option. This is particularly true for patients presenting with IPF, and it is 1 of the most common indications for lung transplantation. Patients with respiratory failure and ILD should be evaluated early in the hospital course for transplantation or considered for transfer to a transplant center. General contraindications to transplant are age older than 70 years, underweight or elevated BMI (generally higher than 30 ), malignancy within the last 2 years (with the exception of cutaneous squamous and basal cell tumors), untreatable major organ dysfunction other than the lung, noncurable chronic extrapulmonary infection (chronic active viral hepatitis $B$, hepatitis $C$, human immunodeficiency virus), significant chest wall deformity, untreatable psychiatric or psychologic disease, substance addiction within the last 6 months, or lack of dependable social support. ${ }^{40}$ In select patients with ILD and gas exchange abnormalities, mechanical ventilation or extracorporeal membrane oxygenation may be used to bridge a patient to lung transplantation. ${ }^{41}$

\section{What should you tell your ILD patient to expect at discharge?}

Accurate diagnosis is important not only for acute inpatient management, but for informing long-term prognosis. Acute-onset ILD tends to be more reversible, to be responsive to medical therapy, and to have a more favorable overall outcome. On the other hand, acute exacerbations of established ILD, particularly IPF, can have a more unfavorable and treatment-refractory course. Once a diagnosis is established, it is important both to provide patients with information and ensure appropriate outpatient follow-up. The Pulmonary Fibrosis Foundation (the largest U.S. advocacy and support organization for patients 
with ILD) provides information on ILD to patients and families and can serve as an important educational source. ${ }^{42}$ Prior to discharge, it is important to evaluate the oxygen needs of patients at rest and with exertion. Referral to an ILD center at discharge is important whenever possible, to monitor clinical symptoms and lung function, initiate or assess response to treatment, and provide supportive care, including oxygen therapy, pulmonary rehabilitation, and outpatient lung transplant referral.

\section{CONCLUSION}

ILD is a group of heterogeneous disorders characterized by lung inflammation and fibrosis. Although the onset of disease is typically insidious, patients can present acutely requiring

\section{References}

1. Travis WD, Costabel U, Hansell DM, et al. An official American Thoracic Society/ European Respiratory Society statement: Update of the international multidisciplinary classification of the idiopathic interstitial pneumonias. Am J Respir Crit Care Med. 2013;188(6):733-748.

2. Kim DS. Acute exacerbation of idiopathic pulmonary fibrosis. Clin Chest Med. 2012;33(1):59-68

3. Collard HR, Ryerson CJ, Corte TJ, et al. Acute Exacerbation of Idiopathic Pulmonary Fibrosis. An International Working Group Report. Am J Respir Crit Care Med. 2016;194(3):265-275

4. King TE Jr, Tooze JA, Schwarz MI, Brown KR, Cherniack RM. Predicting survival in idiopathic pulmonary fibrosis: scoring system and survival model. Am J Respir Crit Care Med. 2001;164(7):1171-1181.

5. Behr J. Approach to the diagnosis of interstitial lung disease. Clin Chest Med. 2012;33(1):1-10.

6. Raghu G, Brown KK. Interstitial lung disease: clinical evaluation and keys to an accurate diagnosis. Clin Chest Med. 2004;25(3):409-419.

7. Bradley B, Branley HM, Egan JJ, et al. Interstitial lung disease guideline: the British Thoracic Society in collaboration with the Thoracic Society of Australia and New Zealand and the Irish Thoracic Society. Thorax. 2008;63(suppl 5):v1-v58.

8. Cooper JA Jr, White DA, Matthay RA. Drug-induced pulmonary disease. Part 1: Cytotoxic drugs. Am Rev Respir Dis. 1986;133(2):321-340.

9. Cooper JA Jr, White DA, Matthay RA. Drug-induced pulmonary disease. Part 2. Noncytotoxic drugs. Am Rev Respir Dis. 1986;133(3):488-505.

10. Diffuse Lung Disease Questionnaire for Patients. Available at: https://www.chestnet.org/ /media/chesnetorg/Foundation/Documents/Lung Disease Questionaire. ashx. Accessed August 15, 2016.

11. Pipavath S, Godwin JD. Imaging of interstitial lung disease. Clin Chest Med. 2004:25(3):455-465, v-vi.

12. Fujimoto K, Taniguchi H, Johkoh T, et al. Acute exacerbation of idiopathic pulmonary fibrosis: high-resolution CT scores predict mortality. Eur Radiol. 2012;22(1):83-92.

13. Mayo JR. CT evaluation of diffuse infiltrative lung disease: dose considerations and optimal technique. J Thorac Imaging. 2009;24(4):252-259.

14. Allen JN, Pacht ER, Gadek JE, Davis WB. Acute eosinophilic pneumonia as a reversible cause of noninfectious respiratory failure. New Engl J Med. 1989;321(9):569-574

15. Selman M, Pardo A, King TE Jr. Hypersensitivity pneumonitis: insights in diagnosis and pathobiology. Am J Respir Crit Care Med. 2012;186(4):314-324.

16. Churg A, Schwarz M. Transbronchial biopsy and usual interstitial pneumonia: a new paradigm? Chest. 2006;129(5):1117-1118.

17. Shim HS, Park MS, Park IK. Histopathologic findings of transbronchial biopsy in usual interstitial pneumonia. Pathol Int. 2010;60(5):373-377.

18. Raghu G, Collard HR, Egan JJ, et al. An official ATS/ERS/JRS/ALAT statement: idiopathic pulmonary fibrosis: evidence-based guidelines for diagnosis and management. Am J Respir Crit Care Med. 2011;183(6):788-824.

19. Carmona EM, Kalra S, Ryu JH. Pulmonary sarcoidosis: diagnosis and treatment. Mayo Clin Proc. 2016;91(7):946-954

20. Tomassetti S, Wells AU, Costabel U, et al. Bronchoscopic lung cryobiopsy increases diagnostic confidence in the multidisciplinary diagnosis of idiopathic pulmonary fibrosis. Am J Respir Crit Care Med. 2016;193(7):745-752.

21. Johannson KA, Marcoux VS, Ronksley PE, Ryerson CJ. Diagnostic yield and complications of transbronchial lung cryobiopsy for interstitial lung disease. A systematic review and metaanalysis. Ann Am Thorac Soc. 2016;13(10):1828-1838.

22. Patel NM, Borczuk AC, Lederer DJ. Cryobiopsy in the diagnosis of interstitial lung hospitalization. Inpatient management varies significantly depending on ILD subtype, and, therefore, accurate diagnosis is key in determining treatment and prognosis. As we develop an improved understanding of the mechanisms of acute presentations of ILD, and our approaches to detection and treatment improve as a result of clinical trials, we anticipate continued modifications to this shared framework.

Disclosure: Dr. Collard reports personal fees from Alkermes, aTyr Pharmaceuticals, Bayer, Boehringer Ingelheim, Bristol-Myers Squibb, Global Blood Therapeutics, Genoa, ImmuneWorks, Moerae Matrix, Navitor, Parexel, Patara, Pharma Capital Partners, Prometic, Takeda, Toray, and Xfibra, outside the submitted work. Drs. Farrand and Shah report no financial conflicts of interest.

disease. A step forward or back? Am J Respir Crit Care Med. 2016;193(7):707-709.

23. Rishi Raj M, Kirtee Raparia, MD, David A. Lynch, MD, Kevin K. Brown, MD. Surgical lung biopsy for interstitial lung diseases. Chest. 2016.

24. Kreider ME, Hansen-Flaschen J, Ahmad NN, et al. Complications of video-assisted thoracoscopic lung biopsy in patients with interstitial lung disease. AnnAm Thor Surg. 2007;83(3):1140-1144

25. Churg A, Wright JL, Tazelaar HD. Acute exacerbations of fibrotic interstitial lung disease. Histopathology. 2011;58(4):525-530.

26. Churg A, Muller NL, Silva CI, Wright JL. Acute exacerbation (acute lung injury of unknown cause) in UIP and other forms of fibrotic interstitial pneumonias. Am J Surg Pathol. 2007;31(2):277-284.

27. Jones KD, Urisman A. Histopathologic approach to the surgical lung biopsy in interstitial lung disease. Clin Chest Med. 2012;33(1):27-40.

28. Hutchinson JP, Fogarty AW, McKeever TM, Hubbard RB. In-hospital mortality after surgical lung biopsy for interstitial lung disease in the United States. 2000 to 2011. Am J Respir Crit Care Med. 2016;193(10):1161-1167.

29. Rhee CK, Min KH, Yim NY, et al. Clinical characteristics and corticosteroid treatment of acute eosinophilic pneumonia. Eur Respir J. 2013;41(2):402-409.

30. Lazor R, Vandevenne A, Pelletier A, Leclerc P, Court-Fortune I, Cordier JF. Cryptogenic organizing pneumonia. Characteristics of relapses in a series of 48 patients. The Groupe d'Etudes et de Recherche sur les Maladles "Orphelines" Pulmonaires (GERM"O”P). Am J Respir Crit Care Med. 2000;162(2 Pt 1):571-577.

31. Richeldi L, Costabel U, Selman M, et al. Efficacy of a tyrosine kinase inhibitor in idiopathic pulmonary fibrosis. New Engl J Med. 2011;365(12):1079-1087.

32. Richeldi L, du Bois RM, Raghu G, et al. Efficacy and safety of nintedanib in idiopathic pulmonary fibrosis. New Engl J Med. 2014;370(22):2071-2082.

33. Azuma A, Nukiwa T, Tsuboi E, et al. Double-blind, placebo-controlled trial of pirfenidone in patients with idiopathic pulmonary fibrosis. Am J Respir Crit Care Med. 2005;171(9):1040-1047.

34. Noble PW, Albera C, Bradford WZ, et al. Pirfenidone in patients with idiopathic pulmonary fibrosis (CAPACITY): two randomised trials. Lancet. 2011;377(9779):1760-1769.

35. King TE Jr, Bradford WZ, Castro-Bernardini S, et al. A phase 3 trial of pirfenidone in patients with idiopathic pulmonary fibrosis. New Engl J Med. 2014;370(22): 2083-2092.

36. Saydain G, Islam A, Afessa B, Ryu JH, Scott JP, Peters SG. Outcome of patients with idiopathic pulmonary fibrosis admitted to the intensive care unit. Am J Respir Crit Care Med. 2002;166(6):839-842

37. Vianello A, Arcaro G, Battistella L, et al. Noninvasive ventilation in the event of acute respiratory failure in patients with idiopathic pulmonary fibrosis. J Crit Care. 2014;29(4):562-567

38. Blivet S, Philit F, Sab JM, et al. Outcome of patients with idiopathic pulmonary fibrosis admitted to the ICU for respiratory failure. Chest. 2001;120(1):209-212.

39. Mollica C, Paone G, Conti V, et al. Mechanical ventilation in patients with endstage idiopathic pulmonary fibrosis. Respiration. 2010;79(3):209-215

40. Orens JB, Estenne M, Arcasoy S, et al. International guidelines for the selection of lung transplant candidates: 2006 update--a consensus report from the Pulmonary Scientific Council of the International Society for Heart and Lung Transplantation. J Heart Lung Transplant. 2006;25(7):745-755

41. Hoopes CW, Kukreja J, Golden J, Davenport DL, Diaz-Guzman E, Zwischenberger JB. Extracorporeal membrane oxygenation as a bridge to pulmonary transplantation. J Thorac Cardiovasc Surg. 2013;145(3):862-867; discussion 867-868.

42. Pulmonary Fibrosis Foundation. http://pulmonaryfibrosis.org/. Accessed August 31, 2016 